\title{
review
}

\section{Dynamic contrast-enhanced ultrasound of the bowel wall with quantitative assessment of Crohn's disease activity in childhood}

\author{
Damjana Kljucevsek¹, Dubravka Vidmar², Darja Urlep, Rok Dezman² \\ ${ }^{1}$ Radiology Unit, Children's Hospital, University Medical Centre Ljubljana, Slovenia \\ ${ }^{2}$ Institute of Radiology, University Medical Centre Ljubljana, Slovenia \\ ${ }^{3}$ Department of Gastroenterology, Hepatology and Nutrition, Children's Hospital, University Medical Centre Ljubljana, \\ Slovenia
}

Radiol Oncol 2016; 50(4): 347-354.

Received 5 March 2015

Accepted 11 June 2015

Correspondence to: Damjana Ključevšek, M.D., Radiology Unit, Children's Hospital, University Medical Centre Ljubljana, Bohoričeva 20, SI-1000 Ljubljana, Slovenia. Phone: +386 152292 64; Fax: +386 1522 29 93; E-mail: damjana.kljucevsek@gmail.com

Disclosure: No potential conflicts of interest were disclosed.

Background. Contrast-enhanced ultrasound (CEUS) has become an established non-invasive, patient-friendly imaging technique which improves the characterization of lesions. In addition, dynamic contrast-enhanced ultrasound (DCE-US) provides valuable information concerning perfusion of examined organs. This review addresses current applications of CEUS in children, focused on DCE-US of the bowel wall in patients with Crohn disease, which enables realtime assessment of the bowel wall vascularity with semi-quantitative and quantitative assessment of disease activity and response to medical treatment.

Conclusions. Crohn's disease is a chronic inflammatory relapsing disease. Frequent imaging re-evaluation is necessary. Therefore, imaging should be as little invasive as possible, children friendly with high diagnostic accuracy. US with wide varieties of techniques, including CEUS/DCE-US, can provide an important contribution for diagnosing and monitoring a disease activity. Even if the use of US contrast agent is off-label in children, it is welcome and widely accepted for intravesical use, and a little less for intravenous use, manly in evaluation of parenchymal lesions. To our knowledge this is the first time that the use of DCE-US in the evaluation of activity of small bowel Crohn disease with quantitative assessment of kinetic parameters is being described in children. Even if the results of the value and accuracy of different quantitative kinetic parameters in published studies in adult population often contradict one another there is a great potential of DCE-US to become a part of the entire sonographic evaluation not only in adults, but also in children. Further control studies should be performed.

Key words: contrast-enhanced ultrasound (CEUS); dynamic contrast-enhanced ultrasound (DCE-US); Crohn disease; quantification; children

\section{Introduction}

Contrast-enhanced ultrasound (CEUS) has been established as a valuable tool in many clinical applications. CEUS has improved the detection and characterization of different lesions in comparison to conventional ultrasound (US). The increasing role of CEUS is based on widespread availability of US equipment with available commercial contrast specific technique on the market, and commercial- ly available safe ultrasound contrast agent (UCA) with no serious adverse effects. The use of CEUS is increasing, particularly in the abdominal US in adults in the diagnosis, differential diagnosis and follow-up in patients with focal liver lesions. The European Federation of Societies for Ultrasound in Medicine and Biology (EFSUMB) has published guidelines and recommendations for the clinical practice of CEUS on hepatic and also on non-hepatic applications in adults. ${ }^{1,2}$ UCA is licensed only for 
cardiac, liver, breast and vascular applications, but this is not an obstacle for a wider use in many other clinical applications..$^{2,3}$ Dynamic contrast-enhanced ultrasound (DCE-US) is a step forward in the quantification of tissue/lesion enhancement and tissue/ lesion perfusion.

The second-generation UCA has not been registered for individuals younger than 18 years of age which makes the clinical application of UCA even harder. ${ }^{4}$ The possibility of a fast, accurate and safe diagnosis of many diseases is thus significantly reduced even if safety considerations of UCA are most promising compared to iodine or paramagnetic contrast agent. $5-8$ "Pediatric CEUS Data Registry" has been established on the EFSUMB website, which allows data input in a prospective manner and records any adverse events. ${ }^{9}$ The main advantages of CEUS in childhood are that this is a radiation-free method, that there is no need for anaesthesia or sedation in small children and that it is easily performed in children because of a smaller body size and more favourable tissue composition compared to adults. ${ }^{4}$ UCA is widely used in children for intravesical application to perform echoenhanced voiding urosonography, an established method for vesicoureteric reflux evaluation. .0,11 $^{10}$ Some papers have been published describing CEUS examination with intravenous application in the paediatric population after blunt injuries of the abdominal cavity (liver and spleen injuries) and for assessment, characterization, and monitoring of parenchymal lesions in childhood. ${ }^{12-16}$ Metaanalysis of literature connected with CEUS performed in children was done.,17 To our knowledge, so far no report of CEUS/DCE-US examinations of the bowel wall in children with Crohn's disease has been published and no study has been done or published study that would evaluate DCE-US of the bowel wall with quantitative assessment of Crohn's disease activity or monitor medical therapy in children. However, DCE-US presents a huge step forward in combining morphologic and functional information regarding the disease activity and response to therapy.

\section{Evaluation of inflammatory activity in Crohn's disease}

Crohn's disease is a chronic inflammatory disease of the gastrointestinal tract. An important characteristic of this condition is that the episodes of inflammation alternate with the periods of remission. Therefore, frequent re-evaluation of the inflammatory activity to plan a proper therapy is necessary in many patients. There are some established methods for the evaluation of paediatrics Crohn's disease activity in children. A gold standard in the assessment of the degree of the Crohn's disease is upper and lower endoscopy (endoscopic severity score) with biopsy, but it is considered an invasive method, which also requires anaesthesia or deep sedation in paediatric patients. There are several surrogate markers for the disease activity that clinicians have traditionally used, including the clinical paediatric Crohn's disease activity index (PCDAI) and laboratory markers of inflammation C-reactive protein (CRP), and more recently faecal markers such as faecal calprotectin. All of these markers, however, have limitations. ${ }^{18}$ Various imaging methods like US with colour and pulsed Doppler, CT and MR enterography play a significant role in the evaluation of the disease activity. No significant differences in diagnostic accuracy among the imaging techniques were observed. ${ }^{19,20}$ In every day clinical praxis the determination of the activity depends on the results given by the various complementary markers and exams depending on local abilities.

In general, the main goal in paediatric patients with Crohn's disease is to find an accurate noninvasive method for the assessment of the disease activity, which is simple, quickly performed, widely available, and well tolerated by patients. High-resolution bowel US is an important imaging technique for the diagnosis and the follow-up of children with Crohn's disease. It can evaluate the localization and the length of the affected intestinal segments, and identify intra-abdominal complications. Doppler techniques can visualize and semi-quantify the bowel wall vascularization. ${ }^{21-24}$ However, DCE-US of the bowel wall with the intravenous administration of the second-generation UCA has all the potentials to become the new technique because it allows real-time examination of the bowel wall perfusion and microvascularization, and enables an objective quantitative measurement of the enhancement by analysing the parameters of the time-intensity curve (TIC)..$^{25-28}$

\section{Dynamic contrast-enhanced ultrasound of the bowel wall with quantitative assessment of Crohn's disease activity}

\section{Diagnostic value of CEUS and DCE-US of the bowel wall: review of the published articles}

CEUS of the bowel wall was introduced to evaluate the intestinal wall hyperaemia and the bowel 
wall microvascularization. An early pathological change in patients with active Crohn's disease is neovascularization of the bowel wall, characterized by the development of new capillary vessels in the lamina propria and submucosa. Low velocity flows in small vessels can be identified now due to advanced techniques using low-mechanicalindex real-time harmonic sonography associated with the second-generation UCAs, which are more stable and remain for a longer period in the intestinal wall microcirculation. Due to the small size microbubbles $(1-7 \mu \mathrm{m})$ the use of UCA allows the assessment of the microcirculation in micro-vessels and capillaries, where the vessels have very small diameters, as small as 40 microns. The spatial resolution of CEUS is $0.2-2 \mathrm{~mm} .{ }^{29}$ CEUS thus allows an accurate mapping of the bowel vasculature and perfusion, and it was proved to be superior to conventional colour or power Doppler imaging. ${ }^{30}$

The first studies for the evaluation of the bowel wall perfusion with CEUS were performed in the early 2000s by the first-generation US contrast agent Levovist (Schering, Berlin, Germany). ${ }^{31}$ For the qualitative evaluation of the inflammatory activity in Crohn's disease four different types of perfusion enhancement patterns of the thickened bowel wall are described, including low or absent enhancement (in comparison with the adjacent mesentery), a prevalent submucosal enhancement sparing the muscularis propria, and complete transmural enhancement, either outward (centrifugal), starting from mucosa, or inward (centripetal), starting form perivisceral vessels. Serra et al. found that patients with complete enhancement of the bowel wall or enhancement of the inner layers had high sensitivity and specificity $81 \%$ and $63 \%$ in distinguishing active and inactive disease according to the Crohn disease activity index (CDAI). ${ }^{32}$ The same study performed by Migaleddu et al. has shown even better results (sensitivity and specificity of $93.5 \%$ and $93.7 \%$, respectively). ${ }^{33}$ On the other hand, enhancement patterns are not useful in differentiating responders from non-responders to medical treatment among patients with Crohn's disease. $^{34,35} \mathrm{In}$ fact, the degree of contrast-enhancement is dependent on several confounding factors, such as contrast agent and equipment used, depth of lesion and shadowing from air, or arteries filled with contrast. Inter-observer variability needs to be taken into account.

The next step of CEUS is DCE-US which enables the quantification of the disease activity by quantitative assessment of the bowel wall enhancement. Katzer et al. introduced the quantification of the bowel wall enhancement by using wide band harmonic imaging US and the HDI-Lab software, but there were no correlations between the enhancement and clinical and/or laboratory indices of the disease activity. ${ }^{36}$ At the moment, different softwares for enhancement quantification are available on the market. One is the quantitative analysis of the brightness in regions of the interest localized in the most echogenic zone of the intestinal wall. The software automatically obtained a brightness-time curve, which correlated well with the disease activity found on endoscopy and MRI of the small bowel. ${ }^{37,38}$ Other softwares are used to draw a TIC, which displays the average intensity of UCA in a region of interest as a function of time, reflecting its transit. The analysis of the TIC of the bowel wall offered various kinetic parameters. ${ }^{39}$ The time-to-peak (the time from zero intensity to maximum intensity enhancement of the bowel wall), peak intensity (maximum value of intensity in arbitrary units), the slope of the first ascending tract of the curve (which correlates with time-to-peak enhancement and enhancement velocity), and the area under the TIC are the most promising in the evaluation of the disease activity. However, the results of published studies evaluating the capabilities of DCE-US, using different parameters of quantitative analysis of TIC to classify Crohn's disease activity and response to medical treatment compared to clinical activity index, laboratory inflammation markers, histopathologic analysis, endoscopy, contrast-enhance CT or MR enterography, often contradict one another. Girlich et al. found that a shorter time-to-peak in patients with higher peak enhancement corresponds well with the higher disease activity. ${ }^{40}$ Romanini et al. compared various parameters obtained from TIC analysis during DCE-US to vascular density in biopsy specimens obtained during colonoscopy of the same bowel segment: a higher peak enhancement, a shorter time-to-peak enhancement, a high regional blood flow and a regional blood volume correlate well with a high vascular density in biopsy specimens. ${ }^{41}$ De Franco et al. compared DCEUS parameters to the clinical and endoscopic score for Crohn's disease, and found sensitivity for detecting active Crohn's disease of $97 \%$ for maximum peak intensity and $86 \%$ for wash-in slope coefficient. ${ }^{42}$ Białecki et al. compared parameters of TIC with a low-dose CT enterography and CDAI and found a good correlation between Crohn's disease activity in DCE-US and low-dose CT enterography. ${ }^{43}$ According to Giangregorio et al. vascular activity cannot be simply correlated with clinical 


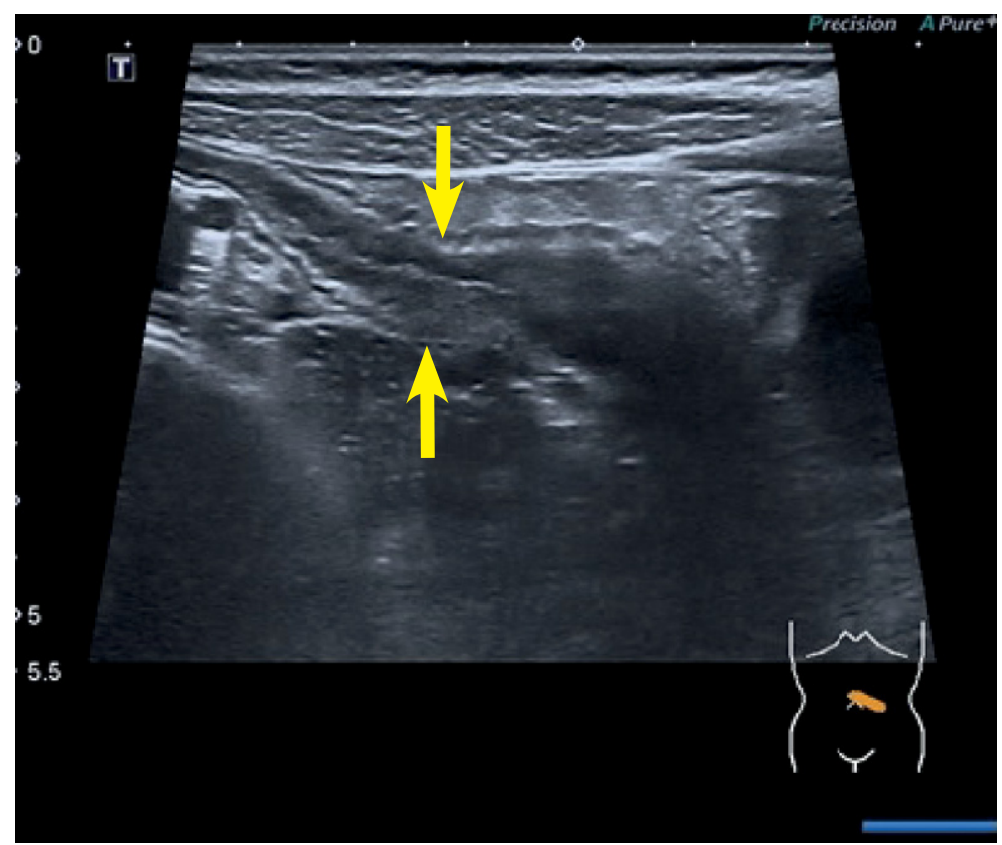

FIGURE 1. Ultrasound (US) of a jejunal segment with a thickened bowel wall.

activity, but its measurement is useful in clinical practice. ${ }^{35}$ It was revealed that in the subgroup of patients with clinically inactive disease and with wall vascularization typical of inflammation, clinically relevant recurrence is present in near future. On the other hand, Wong et al. showed no significant association of TIC analysis (area under curve, time-to-peak peak intensity) with the endoscopic activity. ${ }^{44}$ Girlich et al. performed a quantitative assessment of bowel wall vascularization in healthy volunteers and patients with acute exacerbation of proven Crohn's disease. ${ }^{45}$ Patients had a significantly higher peak enhancement values and regional blood volume than volunteers, but the timeto-peak was significantly shorter in volunteers due to their low enhancement peak. The quantitative parameters should therefore be evaluated together, not individually.

The evaluation of the response to medical treatment with DCE-US provides even more contradictory results. The slope of the first ascending tract of the curve and the area under the TIC were significantly lower while the time-to-peak enhancement was significantly higher after the treatment with a significant correlation with CDAI score. ${ }^{34}$ The last study by the same author contradicts the first one at some point and shows the area under the TIC as the only parameter to distinguish responders from non-responders. ${ }^{46}$ Both of these studies have an important flaw; they were performed with video data that are a log-compressed version of the actual ultrasound intensities, which is not mathematically correct. Studies suggest that log-compression distorts the data and that only linear data of the actual ultrasound intensities (so-called "raw data") should be used for analysis. ${ }^{47}$ Saevik et al. found a significant difference between groups for peak contrast enhancement, rate of wash-in and washout and the area under the TIC in wash-in phase at the examination 1 month after the start of the treatment, but there was no significant difference at any point in the time-related variables between the responders and non-responders groups. ${ }^{48}$

The discordant results of the studies may be due to the different methods used to collect the images (from raw data or from DICOM images) and different softwares available on the market to calculate the parameters of TIC. Even the definitions of some parameters are different using different software packages: i.e. TTP is defined as the time from i.v. administration of contrast medium to maximum enhancement of bowel wall, or time from first appearance of contrast medium in the bowel wall to its maximum enhancement. Therefore, there is no accepted agree about the normal value rangers of TIC parameters. Standardization of software programs should be obtained and further controlled comparable studies should be conducted to determine the real accuracy of the quantitative parameters.

\section{DCE-US protocol}

DCE-US protocol for the assessment of bowel wall vascularization is relatively simple. The adaption of US machine with contrast specific software and the software program for DCE-US quantification is necessary. In addition, software for the TIC evaluation is needed for the qualitative/quantitative evaluation of the bowel wall enhancement on workstation. ${ }^{39}$ There is no need for patient preparation, only fasting for at least 6 hours before examination to ensure standard conditions during investigation. First, accurate baseline US and Doppler examination of the intestine are performed. One or more affected intestinal segments with thickened wall $(\geq 3 \mathrm{~mm})$ are chosen. ${ }^{49} \mathrm{~A}$ high frequency transducer (7.5 to $12 \mathrm{MHz}$ ) is used and it should be kept as still as possible on the selected image of the intestinal segment. Contrast specific software with low mechanical index $(\mathrm{MI}<0.10)$ is switched on. The gain setting is adapted to obtain an anechoic bowel wall. The patient is asked to breathe shallow or to hold the breath during examination. The examination of each examined bowel segment is recorded, starting at the time 
of the intravenous administration in a dose of the second-generation UCA SonoVue (Bracco, Italy), followed by $10 \mathrm{ml}$ of normal saline solution $(0.9 \%$ $\mathrm{NaCl})$. The dose of UCA for SonoVue varies from $1.2 \mathrm{ml}$ in case of low-frequency transducers to 2.4 to $4.8 \mathrm{ml}$ in case of high-frequency transducers per cycle with maximal doze of $10 \mathrm{ml}$ in adults, and it depends also on the sensitivity of the equipment used. ${ }^{28}$ The dose of UCA in children can be calculated according to the formula: dose $(\mathrm{ml})=$ child age (years) / 10, but not less than $0.1 \mathrm{ml}$ per single application. ${ }^{49}$ Continuous imaging is performed for 60 - 90 seconds. The examination is stored in the scanner as a raw data video clip. One or more regions of interest (ROI) are drawn encompassing the thickened anterior wall, excluding the lumen and perivisceral tissues. In postprocesing the raw data is sent to the workstation by one of the software packages, either to draw a TIC and automatically calculate various kinetic quantitative parameters as described above, or to draw a brightness time curve using quantitative analysis of the brightness in ROI, and to calculate the percentage of the increase in the wall brightness.

\section{Clinical indications, advantages and limitation of CEUS/DCE-US of the bowel wall}

According to EFSUMB guidelines and recommendations on the clinical practice of CEUS/DCE-US considering the gastrointestinal tract CEUS/DCEUS is recommended when estimating the disease activity in inflammatory bowel disease, when discerning between fibrous and inflammatory strictures in Crohn's disease, when characterizing the suspected abscesses, and when confirming and following the route of fistulas. ${ }^{4}$ Even if the studies contradict one another to a certain degree, as described above the DCE-US with the assessment of the quantitative results might be able to differentiate between responders and non-responders to the treatment in patients with Crohn's disease, and thus to improve therapy planning and monitoring of the efficacy of the treatment, together with other non-invasive inflammatory markers and imaging examinations. To determine the most valuable parameters of TIC, further controlled and comparable studies should be conducted. However, we should be aware that there are no absolute numbers and that quantitative parameters should be analysed as a whole. A comparison of the parameters is mandatory in follow-up studies monitoring the therapy.

The main advantage of CEUS/DCE-US examination of the bowel wall particularly in children is that the examination is non-invasive, radiation-

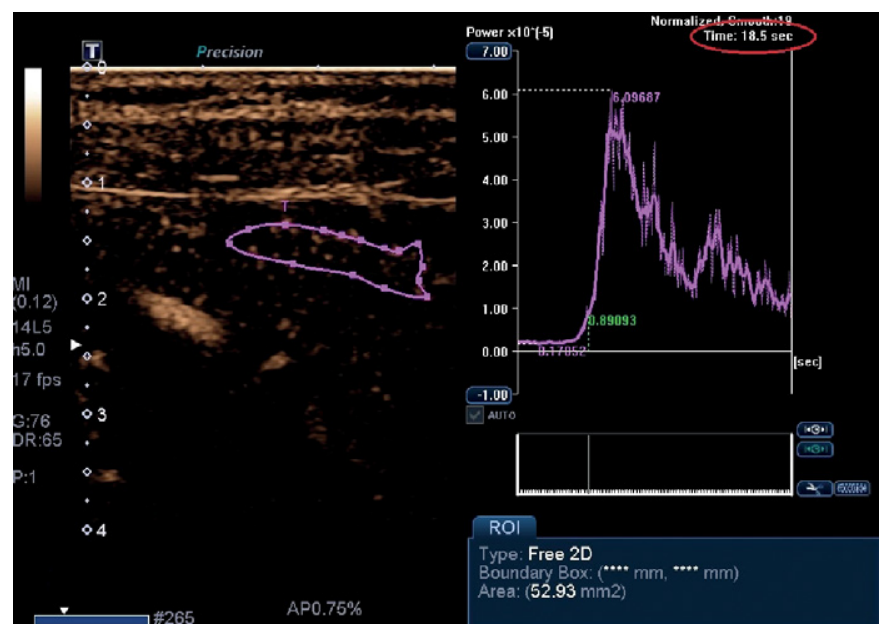

(A)

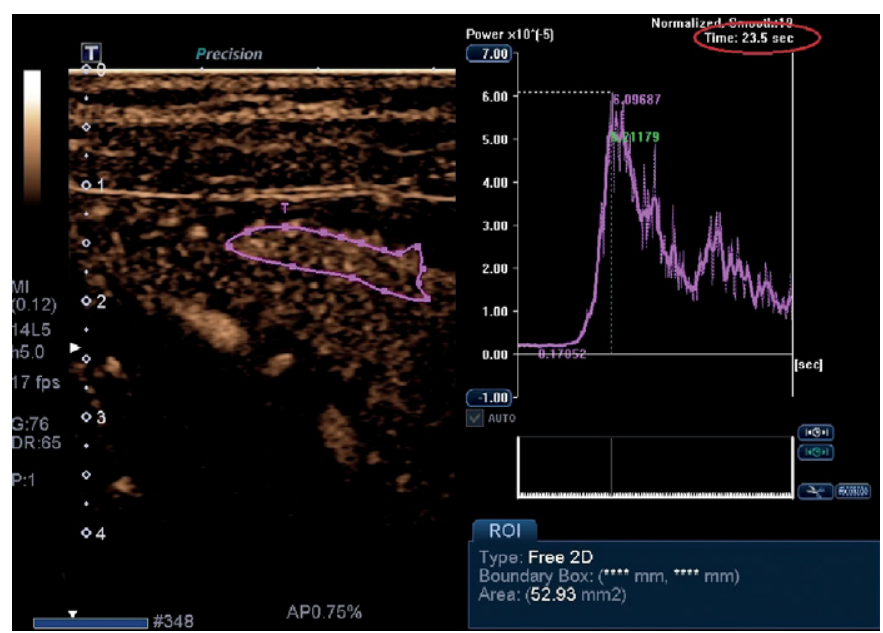

FIGURE 2. Contrast-enhanced ultrasound: the region of interest (ROI) is determined (A) when the contrast medium first appears in the affected bowel wall corresponding to starting point at time-intensity curve, (B) when the maximum enhancement was seen corresponding to peak at time-intensity curve. The calculated time-to-peak is 5 seconds (18.5 sec to 23.5).

free, easy repeatable, patient friendly, very well tolerated and accepted, and it does not require any specific preparation. An additional advantage in children is that CEUS/DCE-US is easily performed because of the smaller body size and more favourable tissue composition compared to adults, and that there is no need for anaesthesia in smaller children. Motion artefacts produced by peristalsis or intestinal contents do not impair CEUS/DCE-US as is the case with colour Doppler or during MR enterography. Even if the ROI cannot be continuously placed over a defined area because of the motion of the intestinal wall, it is possible to analyse the contrast agent enhancement from individual images. ${ }^{25}$ In addition, UCA is considered the safest contrast agent with the lowest rate of adverse effects. ${ }^{5-7}$ 

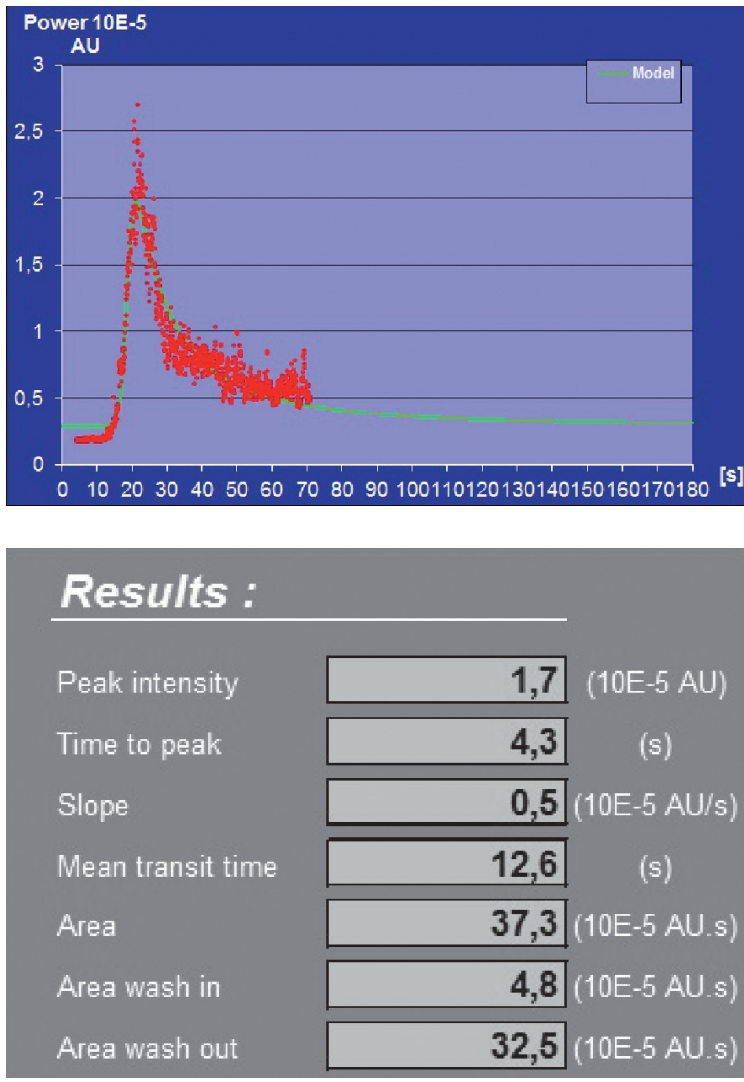

FIGURE 3. Postprocesing results obtained from raw data imported in workstation with dedicated quantification software (Workstation UltraExtend FX with IGR protocol): (A) Time-intensity curve (TIC), and (B) the table of results of the calculated quantitative kinetic parameters from TIC.

The main limitations are the off-label use of UCA in childhood and the evaluation of the limited number of the effected bowel segments seen by the use of on basic examination. It is not possible to evaluate the enhancement if the bowel wall is not clearly identified, or it is too thin (it should be $\geq 3 \mathrm{~mm}$ ). Each bowel segment needs an injection of UCA. DCE-US needs specific software installed on US machine to be able to quantify the enhancement. Postprocesing software packages of different kinds are on the market and a comparison of these programs has not been obtained yet. The selection of the ROI to create TIC also depends on the radiologist and it can be a potential cause of interobserver variability.

In our case a 13-year-old boy with known Crohn's disease was admitted to the hospital because of the acute exacerbation of the disease, presented by vomiting, severe abdominal cramps, weight loss and inappetence. He had been diagnosed with isolated small bowel Crohn's disease two years ago. At the time of the diagnosis remis- sion had been induced by exclusive enteral nutrition and it was well maintained by azathioprine ( $2.5 \mathrm{mg} / \mathrm{kg}$ daily) for 2 years. At the time of the exacerbation, he had elevated laboratory markers (erythrocyte sedimentation rate [ESR] 43, CRP 25) and faecal calprotectin $(310 \mathrm{mg} / \mathrm{kg}$, normal value < $50 \mathrm{mg} / \mathrm{kg}$ of stool). The estimated PCDAI was 37 . The abdominal US showed aperistaltic segments of the small intestine with a thickened wall surrounded by echogenic mesentery and enlarged lymph nodes. The terminal ileum and colon were normal on US. MR enterography confirmed irregular thickened segments of the small bowel wall with entero-enteric fistulas and intense enhancement of the effected bowel wall. Upper endoscopy and ileocolonoscopy showed normal mucosa of the upper gastrointestinal tract, ileum and colon. Anti-TNF $\alpha$ (infliximab) biological treatment was introduced. Control US and control MR enterography showed only a slightly improvement of the inflammation. Laboratory findings were normal, except for the increased calprotectin. Anti-TNF $\alpha$ treatment was continued and the patient was in clinical remission. Three months later he complained of fatigue, inappetence and some weight lost. At that time laboratory markers were normal (normal ESR and CRP), only calprotectin was elevated $(495 \mathrm{mg} / \mathrm{kg})$. Estimated PDCAI was 40.The boy refused to perform control MR enterography for the disease activity evaluation because he felt sick after drinking the contrast medium and had nausea after the application of glucagon. Therefore, DCE-US of the bowel wall was suggested to evaluate its inflammatory activity and a written informed consent by him and his parents was obtained (Figures 1 and 2). The boy was very cooperative during the examination. He tolerated it very well, without any side effects compared to previous MR enterography. The quantitative analyses of TIC suggest according to some literature and our own experiences that there is still a quite active inflammation of jejunum: time-to-peak enhancement was short, less than 5 seconds, the intensity of the enhancement was high (Figure 3). Time-topeak peak intensity (TTP) less than 7 seconds and intense enhancement of the bowel wall are sings for active inflammation of the bowel wall. ${ }^{40}$ According to the clinical condition and the results of DCE-US, anti-TNF $\alpha$ treatment was optimized: the application interval was shortened from 8 to 4 weeks. Five months later control US and the blood inflammatory parameters were normal, PCDAI was below 10 and calprotectin decreased to $56 \mathrm{mg} / \mathrm{kg}$. The boy is doing clinically well now, shows no fatigue and he is gaining weight and height. 
To our knowledge, no study evaluating DCE-US of the bowel wall with quantitative assessment of Crohn's disease activity in children has yet been published. Our case shows the importance of DCEUS as a non-invasive method for children with Crohn's disease, especially during follow-up of the disease activity. In this case the results of DCE-US combined with clinical signs and elevated calprotectin presented the main indicator, which influenced the therapy regime.

\section{Conclusions}

DCE-US is a dynamic real-time examination of the bowel wall perfusion, which can be performed in a short period of time, is inexpensive, easy available compared to MR enterography, and radiation free compared to CT enterography. It is well tolerated by patients, which is very important in chronic patients and children, in whom frequent examinations are necessary. At the moment DCE-US cannot replace other cross-sectional imaging for global bowel assessment, but it has a great potential to become an important part in algorithms for the diagnosis and monitoring of the disease activity in patients with Crohn's disease. However, controlled studies with comparable softwares of TIC evaluation should be performed to establish accuracy of the method and its value in children.

\section{References}

1. Claudon $\mathrm{M}$, Dietrich $\mathrm{BI}$, Choi $\mathrm{BI}$, Cosgrove $\mathrm{DO}$, Kudo $\mathrm{M}$, Nolsøe $\mathrm{CP}$, et al. Guidelines and good clinical practice recommendations for contrastenhanced ultrasound (CEUS) in the liver - update 2012. Ultraschall Med 2013; 34: 11-29.

2. Piscaglia F, Nolsøe C, Dietrich CF, Cosgrove DO, Gilja OH, Bachmann NM, et al. The EFSUMB guidelines and recommendations on the clinical practice of contrast-enhanced ultrasound (CEUS): update 2011 on non-hepatic applications. Ultraschall Med 2012; 33: 33-59.

3. Li X, Liang P, Yu J, Yu XL, Liu FY, Cheng ZG, et al. Role of contrast-enhanced ultrasound in evaluating the efficiency of ultrasound guided percutaneous microwave ablation in patients with renal cell carcinoma. Radiol Oncol 2013; 47: 398-404.

4. Schreiber-Dietrich D, Dietrich CF. Contrast enhanced ultrasound (CEUS) and off-label use (in children). Ultraschall Med 2012; 33: 295-6.

5. Darge K, Papadopoulou F, Ntoulia A, Bulas DI, Coley BD, Fordham LA, et al. Safety of contrast-enhanced ultrasound in children for non-cardiac applications. Pediatr Radiol 2013; 43: 1063-73.

6. Riccabona M. Application of a second-generation US contrast agent in infants and children--a European questionnaire-based survey. Pediatr Radiol 2012; 42: 1471-80

7. Piskunowicz M, Kosiak W, Batko T, Piankowski A, Połczyńska K, AdamkiewiczDrożyńska E. Safety of intravenous application of second-generation ultrasound contrast agent in children: prospective analysis. Ultrasound Med Biol 2015; 41: 1095-9.
8. Piscaglia F, Bolondi L. The safety of SonoVue in abdominal applications: retrospective analysis of 23188 investigations. Ultrasound Med Biol 2006; 32: $1369-75$.

9. Sellars ME, Deganello A, Sidhu PS. Paediatric contrast-enhanced ultrasound (CEUS): a technique that requires co-operation for rapid implementation into clinical practice. Ultraschall Med 2014; 35: 203-6.

10. Piskunowicz M, Kosiak W, Batko T. Intravenous application of secondgeneration ultrasound contrast agents in children: a review of the literature. Ultraschall Med 2012; 33: 135-40.

11. Darge K. Voiding urosonography with US contrast agent for diagnosis of vesicoureteric reflux in children: an update. Pediatr Radiol 2010; 40: 956-62.

12. Darge K, Ntoulia K, Evangelou V, Papadopoulou F. A meta-analysis of the diagnostic performance of contrast enhanced voiding urosonography (ceVUS). Pediatr Radiol 2012; 42 (Suppl): S259-60.

13. Oldenburg A, Hohmann J, Skrok J, Albrecht T. Imaging of pediatric splenic injury with contrast-enhanced ultrasonography. Pediatr Radiol 2004; 34: $351-4$

14. Thorelius L. Emergency real-time contrast-enhanced ultrasonography for detection of solid organ injuries. Eur Radiol 2007; 17(Suppl): F107-11.

15. Valentino M, Serra C, Pavlica P, Labate AM, Lima M, Baroncini S, et al. Blunt abdominal trauma: diagnostic performance of contrast-enhanced ultrasound in children - initial experience. Radiology 2008; 246: 903-9.

16. Jacob J, Deganello A, Sellars ME, Hadzic N, Sidhu PS. Contrast enhanced ultrasound (CEUS) characterization of grey-scale sonographic indeterminate focal liver lesions in pediatric practice. Ultraschall Med 2013; 34: 529-40.

17. Schreiber-Dietrich D, Cui XW, Piscaglia F, Gilja OH, Dietrich CF. Contrast enhanced ultrasound in pediatric patients: a real challenge. $Z$ Gastroenterol 2014; 52: 1178-84.

18. Vilela EG. Evaluation of inflammatory activity in Crohn's disease and ulcerative colitis. World J Gastroenterol 2012; 18: 872-81.

19. Horsthuis K, Bipat S, Bennink RJ, Stoker J. Inflammatory bowel disease diagnosed with US, MR, Scintigraphy, and CT: Metaanalysis of prospective studies. Radiology 2008; 247: 64-79.

20. Schreyer AG, Menzel C, Friderich C, Poschenrieder F, Egger L, Dornia C, et al. Comparison of high-resolution ultrasound and MR-enterography in patients with inflammatory bowel disease. World J Gastroenterol 2011; 17: 1018-25.

21. Spalinger J, Patriquin H, Miron MC, Marx G, Herzog D, Dubois J, et al. Doppler US in patient with Crohn disease: vessel density in the diseased bowel reflects disease activity. Radiology 2000; 217: 787-91.

22. Neye H, Voderholzer W, Rickes S, Weber J, Wermke W, Lochs H. Evaluation of criteria for the activity of Crohn's disease by power Doppler sonography. Dig Dis 2004; 22: 67-72.

23. Sjekavica I, Barbarić-Babić V, Šunjara V, Kralik M, Senečić-Čala I, DujšinM, et al. Resistance index in mural arteries of thickened bowel wall: predictive value for Crohn disease activity assessment in pediatric patients. Wien Klin Wochenschr 2013; 125: 254-60.

24. Allgayer H, Braden B, Dietrich CF. Transabdominal ultrasound in inflammatory bowel disease. Conventional and recently developed techniques - update. Medical Ultrason 2011; 13: 302-13.

25. Ripollés T, Martínez-Pérez MJ, Blanc E, Delgado F,Vizuete J, Paredes JM, et al Contrast-enhanced ultrasound (CEUS) in Crohn's disease: technique, image interpretation and clinical applications. Insights Imaging 2011; 2: 639-52.

26. De Franco A, Marzo M, Felice C, Pugliese D, Veronica AD, Bonomo L, et al. lleal Crohn's disease: CEUS determination of activity. Abdom Imaging 2012; 37: 359-68.

27. Migaleddu V, Quaia E, Scanu D, Carla S, Bertolotto M, Campisi G, et al. Inflammatory activity in Crohn's disease: CE-US. Abdom Imaging 2011; 36: $142-8$.

28. Quaia E. Contrast-enhanced ultrasound of the small bowel in Crohn's disease. Abdom Imaging 2013; 38: 1005-13.

29. Leen E, Averkiou M, Arditi M, Burns P, Bokor D, Gauthier T, et al. Dynamic contrast enhanced ultrasound assessment of the vascular effects of novel therapeutics in early stage trials. Eur Radiol 2012; 22: 1442-50. 
30. Schreyer AG, Finkenzeller T, Gössmann H, Daneschnejad M, Müller-Wille R Schacherer $\mathrm{D}$, et al. Microcirculation and perfusion with contrast enhanced ultrasound (CEUS) in Crohn's disease: first results with linear contrast harmonic imaging (CHI). Clin Hemorheol Microcirc 2008; 40: 143-55.

31. Di Sabation A, Fulle I, Ciccocioppo R, Ricevuti L, Tinozzi FP, Tinozzi S, et al. Doppler enhancement after intravenous Levovist injection in Crohn's disease. Inflamm Bowel Dis 2002; 8: 251-7.

32. Serra C, Menzzi G, Labate AM, Giangregorio F, Gionchetti P, Beltrami M, et al. Ultrasound assessment of vascularization of the thickened terminal ileum wall in Crohn's disease patients using a low-mechanical index real-time scanning technique with a second-generation ultrasound contrast agent. Eur J Radiol 2007; 62: 114-21.

33. Migaleddu V, Scanu AM, Quaia E, Rocca PC, Dore MP, Scanu D, et al. Contrast-enhanced ultrasonographic evaluation of inflammatory activity in Crohn's disease. Gastroenterology 2009; 137: 43-61.

34. Quaia E, Migaleddu V, Batratella E, Pizzolato R, Rossi A, Grotto M, et al. The diagnostic value of small bowel wall vascularity after sulphurhexafluroridefilled microbubble injection in patients with Crohn's disease. Correlation with therapeutic effectiveness of specific anti-inflammatory treatment. Eur I Radiol 2009; 69: 438-44.

35. Giangregorio F, Bertone A, Fanigliulo L, Comparato G, Aragona G, Marinone $M G$, et al. Predictive value of time-intensity curves obtained with contrastenhanced ultrasonography (CEUS) in the follow-up of 30 patients with Crohn's disease. J Ultrasound 2009; 12: 151-9.

36. Kratzer W, Schmidt SA, Mittrach C, Haenle MM, Haenle MM, Mason RA, et al. Contrast-enhanced wideband harmonic imaging ultrasound (SonoVue): A new technique for quantifying bowel wall vascularity in Crohn's disease. Scand J Gastroenterol 2005; 40: 985-91.

37. Ripollés T, Martínez MJ, Paredes JM, Blanc E, Flors L, Delgado F. Crohn disease: correlation of findings at contrast-enhanced US with severity at endoscopy. Radiology 2009; 253: 241-8.

38. Malagò R, D'Onofrio M, Mantovani W, D'Alpaos G, Foti G, Pezzato A et al. Contrast-enhanced ultrasonography (CEUS) vs. MRI of the small bowel in the evaluation of Crohn's disease activity. Radiol Med 2012; 117: 268-81.

39. Dietrich CF, Averkiou MA, Correas JM, Lassau N, Leen E, Piscaglia F. An EFSUMB Introduction into Dynamic Contrast-Enhanced Ultrasound (DCEUS) for Quantification of Tumor Perfusion. Ultraschall Med 2012; 33: 344-51.

40. Girlich C, Schacherer D, Jung EM, Schreyer A, Büttner R. Comparison between a clinical activity index (Harvey-Bradshaw-Index), laboratory inflammation markers and quantitative assessment of bowel wall vascularization by contrast-enhanced ultrasound in Crohn's disease. Eur J Radiol 2012; 81: 1105-9.

41. Romanini L, Passamonti M, Navarria M, Lanzarotto F, Villanacci V, Grazioli $L$, et al. Quantitative analysis of contrast-enhanced ultrasonography of the bowel wall can predict disease activity in inflammatory bowel disease. Eur J Radiol 2014; 83: 1317-23.

42. De Franco A, Di Veronica A, Armuzzi A, Roberto I, Marzo M, De Pascalis B, et al. lleal Crohn disease: Mural microvascularity quantified with contrastenhanced US correlates with disease activity. Radiology 2012; 262: 680-8.

43. Białecki M, Białecka A, Laskowska K, Kłopocka M, Liebert A, Lemanowicz A et al. Contrast-enhanced ultrasonography for the determination of Crohn's disease activity - preliminary experience. Pol J Radiol 2014; 79: 70-4.

44. Wong DD, Forbes GM, Zelesco M, Mason R, Pawlik J, Mendelson RM. Crohn's disease activity: quantitative contrast-enhanced ultrasound assessment. Abdom Imaging 2012; 37: 369-76.

45. Girlich C, Jung EM, lesalnieks I, Schreyer AG, Zorger N, Strauch U. Quantitative assessment of bowel wall vascularization in Crohn's disease with contrast-enhanced ultrasound and perfusion analyses. Clin Hemorheol Microcirc 2009; 43: 141-8.

46. Quaia E, Cabibbo B, De Paoli L, Toscano W, Poillucci G, Assunta Cova M The value of time-intensity curves obtained after microbubble contrast agent injection to discriminate responder from non-responders to antiinflammatory medication among patients with Crohn's disease. Eur Radiol 2013; 23: 1650-9.

47. Peronneau P, Lassau N, Leguerney I, Roche A, Cosgrove D. Contrast ultrasonography: necessity of linear data processing for the quantification of tumor vascularization. Ultraschall Med 2010; 31: 370-8.
48. Saevik F, Nylund K, Hausken T, Ødegaard S, Gilja OH. Bowel perfusion measured with dynamic contrast-enhanced ultrasound predicts treatment outcome in patients with Crohn's disease. Inflamm Bowel Dis 2014; 20: 2029-37.

49. Chiorean L, Schreiber-Dietrich D, Braden B, Cui X, Dietrich CF. Transabdomina ultrasound for standardized measurement of bowel wall thickness in norma children and those with Crohn's disease. Med Ultrason 2014; 16: 319-24.

50. Thorelius L. Contrast-Enhances Ultrasound in low-energy blunt trauma. In Lencioni R, editor. Enhancing the role of ultrasound with contrast. Milan: Springer-Verlag Italia; 2006. p. 199. 